\title{
Topological Models of Columnar Vagueness
}

\author{
Thomas Mormann ${ }^{1}$ (D)
}

Received: 19 January 2019 / Accepted: 23 December 2019

(c) Springer Nature B.V. 2020

\begin{abstract}
This paper intends to further the understanding of the formal properties of (higherorder) vagueness by connecting theories of (higher-order) vagueness with more recent work in topology. First, we provide a "translation" of Bobzien's account of columnar higher-order vagueness into the logic of topological spaces. Since columnar vagueness is an essential ingredient of her solution to the Sorites paradox, a central problem of any theory of vagueness comes into contact with the modern mathematical theory of topology. Second, Rumfitt's recent topological reconstruction of Sainsbury's theory of prototypically defined concepts is shown to lead to the same class of spaces that characterize Bobzien's account of columnar vagueness, namely, weakly scattered spaces. Rumfitt calls these spaces polar spaces. They turn out to be closely related to Gärdenfors' conceptual spaces, which have come to play an ever more important role in cognitive science and related disciplines. Finally, Williamson's "logic of clarity" is explicated in terms of a generalized topology ("locology") that can be considered an alternative to standard topology. Arguably, locology has some conceptual advantages over topology with respect to the conceptualization of a boundary and a borderline. Moreover, in Williamson's logic of clarity, vague concepts with respect to a notion of a locologically inspired notion of a "slim boundary" are (stably) columnar. Thus, Williamson's logic of clarity also exhibits a certain affinity for columnar vagueness. In sum, a topological perspective is useful for a conceptual elucidation and unification of central aspects of a variety of contemporary accounts of vagueness.
\end{abstract}

\section{Introduction}

This paper intends to further the understanding of the formal properties of (higherorder) vagueness by connecting theories of (higher-order) vagueness with more recent work in topology. More precisely, we want to address the following topics. To

Thomas Mormann

thomasarnold.mormann@ehu.eus

1 Department of Logic and Philosophy of Science, University of the Basque Country UPV/EHU, Avenida de Tolosa 70, 20080 Donostia-San Sebastian, Spain 
set the stage, we first offer a "translation" of Bobzien's notion of columnar higherorder vagueness into the logic of topological spaces (cf. Bobzien 2012, 2013, 2015). An essential ingredient for her solution to the Sorites paradox is casting the paradox in the framework of modal logic S4.1. The topological translation shows that S4.1 can be characterized as the logic of weakly scattered spaces. These spaces exhibit a particularly nice behaviour with respect to the concept of a boundary, which suggests that weakly scattered spaces offer a convenient framework for Bobzien's account of columnar boundaries.

The purpose of Rumfitt's work on vagueness presented in The Boundary Stones of Logic (Rumfitt 2015 is similar. Rumfitt also seeks a classical solution to the Sorites paradox in the framework of classical logic. For this purpose, he relies on regular open, non-classical semantics. More precisely, he offers a formally precise topological reconstruction of Sainsbury's theory of vague, prototypically defined concepts. This investigation leads him to the class of polar topological spaces, which are just those spaces that characterize modal logic S4.1 used by Bobzien to cope with the Sorites paradox, namely, weakly scattered spaces.

Third, we address Williamson's “logic of clarity” (cf. Williamson 1994, 1999), the formal structure of which can be elucidated in terms of a generalized topology that Breysse and De Glas presented some years ago as an alternative to standard topology under the name "locology" (cf. Breysse and de Glas 2007). Arguably, locology has some conceptual advantages over standard topology with respect to the conceptualization of the notions of boundary and borderline. Moreover, in Williamson's logic of clarity, vague concepts turn out to be almost columnar in the sense of Bobzien. More precisely, a natural notion of a "slim boundary" for the concepts of "fixed-margin models" of the logic of clarity also presents stable columnar vagueness.

The touchstone for every theory of vagueness is whether it contributes to a solution to the Sorites paradox. Vague concepts may be characterized as concepts that possess borderline cases. According to Bobzien, one may distinguish between two kinds of higher-order vagueness: hierarchical higher-order vagueness and columnar higher-order vagueness:

Hierarchical higher-order vagueness is characterized by a hierarchy of consecutively higher orders of borderline cases of a vague predicate (i) that include clear (definite, determinate) borderline cases, and (ii) whose extensions do not overlap... Columnar higher-order vagueness differs from hierarchical higherorder vagueness in that, extensionally, it contains just one kind of borderline cases, and each borderline case is radically higher order, or radically borderline, i.e., borderline borderline..., ad infinitum (Bobzien 2015, 63).

Beyond the class of weakly scattered spaces, many subsets of topological spaces in general exhibit columnar vagueness. It can even be proven that for all topological spaces, all subsets exhibit a concept of stable columnar vagueness that is only slightly weaker than Bobzien's original version of columnar vagueness and suffices to defuse the suspicion that higher-order vagueness is an inconsistent notion. 
The topological explication of Bobzien's concept of columnar vagueness is not only interesting in itself but also relevant for the topological account of vagueness recently proposed in Rumfitt (2015). In this book, the author's main intention concerning problems of vagueness is to offer a new "classical" solution to the Sorites paradox by relying on Mark Sainsbury's influential idea about characterizing vague concepts. According to Sainsbury, concepts are "boundaryless" (cf. Sainsbury 1990; Wright 2007; Rumfitt 2015). Rumfitt offers a precise topological reconstruction of Sainsbury's informal approach. Perhaps surprisingly, Rumfitt's reconstruction leads to the same class of topological spaces that were already shown in Bobzien's, namely, weakly scattered spaces.

The general result of this paper is that for the apparently different accounts of Bobzien, Rumfitt, and Williamson, the resulting concepts of vagueness turn out to be columnar (or stably columnar, in a sense to be rendered precise). This holds for Bobzien's and Rumfitt's accounts, which may be cast in a proper topological framework, and for Williamson's logic of clarity for which generalized topology may be more adequate. For all, higher-order vagueness turns out to be (stably) columnar. In sum, the topological perspective offered in this paper may be useful for the conceptual elucidation and unification of a variety of contemporary accounts of vagueness.

The outline of this paper is as follows: In Sect. 2, we recall some basic concepts and results from topology that are necessary for understanding the subsequent sections. As an application of the topological apparatus, Sect. 3 shows that the class of weakly scattered spaces provides an appropriate class of topological models for dealing with columnar vagueness. Section 4 shows that weakly scattered spaces also provide the appropriate framework for a topological elucidation of how vague concepts may be defined by prototypical or paradigmatic cases. Section 5 addresses Rumfitt's solution to the Sorites paradox, which may be considered "semi-classical" since it is based on classical Boolean logic but uses non-classical regular open semantics. To persuade the reader that polar spaces should not be considered just a more or less contrived device taken from modern mathematics, Sect. 6 shows that the conceptual space approach put forward in cognitive science, linguistics and related sciences provides a rich source for polar spaces in the sense of Rumfitt (cf. Gärdenfors 2000, 2014; Zenker and Gärdenfors 2015). Indeed, conceptual spaces in Gärdenfors' sense give rise to a special type of polar spaces in Rumfitt's sense. As will be explained in detail, the Voronoi tessellation of conceptual space corresponds uniquely to the partition of polar space defined by its regular open concepts.

Section 7 resumes the topic of columnar subsets of arbitrary topological spaces (X, OX). It is proven that this class has the structure of a (non-complete) Boolean algebra with respect to the familiar set-theoretical Boolean operations union, intersection, and complement. Section 8 shows that Williamson's logic of clarity gives rise to a notion of well-behaved stable columnar vagueness, although its defining modal operator $\mathbf{C}^{*}$ does not satisfy the so-called axiom 4 of the modal logic S4. In Sect. 9, we close with some general considerations on the problem of how (for normal modal logics) the alternatives of columnar higher-order vagueness, stable columnar vagueness and "slim" stable columnar vagueness for KTB are to be assessed. 


\section{Basics of Topology}

Let us begin by recalling the basic topological definitions and results that will be used throughout this paper.

Definition 2.1 Let $\mathrm{X}$ be a (non-empty) set. A topological space is a pair (X, OX), where $X$ is a set (of "points") and OX is a family of subsets of X containing X and $\varnothing$ that is closed under finite intersections and arbitrary unions of its elements. The elements of OX are called open sets of space X, and the set-theoretical complements of open sets are called closed sets. The family $\mathrm{OX}$ of open subsets of $\mathrm{X}$ is called a topology on X.

The interior $\operatorname{int}(\mathrm{A})$ of a set $\mathrm{A} \subseteq \mathrm{X}$ is the largest open set contained in $\mathrm{A}$, and the closure $\operatorname{cl}(\mathrm{A})$ of $\mathrm{A}$ is the least closed set containing $\mathrm{A}$. By Definition 2.1, int $\mathrm{A}$ is the union of all open subsets of A. Dually, the closure $\operatorname{cl}(\mathrm{A})$ is the intersection of all closed sets that contain A. Let CA denote the set-theoretical complement of A in X. Then, clearly, int $\mathrm{A}$ and $\mathrm{cl} \mathrm{A}$ are interdefinable: int $\mathrm{A}=\mathrm{C} \mathrm{cl} \mathrm{CA}$ and $\mathrm{clA}=\mathrm{C}$ int $\mathrm{CA}$. For a topological space (X, OX), the interior int and the closure cl can be conceived as operators on the set PX of subsets of X. From the Definition 2.1 of OX, it follows that these operators satisfy the so-called Kuratowski axioms:

Kuratowski Axioms 2.2 Let (X, OX) be a topological space, $\mathrm{A}, \mathrm{B} \subseteq \mathrm{X}$. Then, the operators cl and int satisfy the following axioms.

\begin{tabular}{llll}
\hline (1) & $\operatorname{cl}(\mathrm{A} \cup \mathrm{B})=\operatorname{cl}(\mathrm{A}) \cup \mathrm{cl}(\mathrm{B})$. & $(1)^{*}$ & $\operatorname{int}(\mathrm{A} \cap \mathrm{B})=\operatorname{int}(\mathrm{A}) \cap \operatorname{int}(\mathrm{B})$. \\
$(2)$ & $\operatorname{cl}(\mathrm{cl}(\mathrm{A}))=\operatorname{cl}(\mathrm{A})$. & $(2)^{*}$ & $\operatorname{int}(\operatorname{int}(\mathrm{A}))=\operatorname{int}(\mathrm{A})$. \\
$(3)$ & $\mathrm{A} \subseteq \mathrm{cl}(\mathrm{A})$. & $(3)^{*}$ & $\operatorname{int}(\mathrm{A}) \subseteq \mathrm{A} .(4)$ \\
$(4)$ & $\operatorname{cl}(\varnothing)=\varnothing$. & $(4)^{*}$ & $\operatorname{int}(\mathrm{X})=\mathrm{X}$.
\end{tabular}

Clearly, the axioms (1)-(4) are equivalent to (1)*-(4)*. In the following, we will use these axioms without always explicitly mentioning them.

Definition 2.3 Let $(\mathrm{X}, \mathrm{OX})$ be a topological space, $\mathrm{A}, \mathrm{B} \subseteq \mathrm{X}$.

(i) Set $\mathrm{A}$ is dense iff $\operatorname{cl}(\mathrm{A})=\mathrm{X}$, and set $\mathrm{B}$ is nowhere dense iff int $(\mathrm{cl}(\mathrm{B}))=\varnothing$.

(ii) A point $x \in X$ is isolated iff the singleton $\{x\} \in O X$. The set of isolated points of $\mathrm{X}$ is denoted by $\operatorname{ISO}(\mathrm{X})$.

(iii) $(\mathrm{X}, \mathrm{OX})$ is weakly scattered iff $\operatorname{cl}(\operatorname{ISO}(\mathrm{X}))$ is dense in $\mathrm{X}$, i.e., $\operatorname{cl}(\operatorname{ISO}(\mathrm{X}))=\mathrm{X}$.

For a class $\mathrm{K}$ of topological spaces $(\mathrm{X}, \mathrm{OX})$, let $\mathrm{L}(\mathrm{K})$ denote the set of formulas of the modal propositional calculus that are valid for all members of $\mathrm{K}$ interpreting modal operators $\square$ and $\diamond$ as interior operator int and closure operator cl, 
respectively. Due to a fundamental result of McKinsey and Tarski, $\mathrm{L}(\mathrm{K})$ is a normal extension of S4. Set $\mathrm{L}(\mathrm{K})$ of formulas is called the modal logic of class $\mathrm{K}$ of topological spaces. For instance, let TOP be the class of all topological spaces and ALEX be the class of Alexandroff spaces ${ }^{1}$; then, $\mathrm{L}(\mathrm{TOP})=\mathrm{L}(\mathrm{ALEX})=\mathrm{S} 4$. Many results of this type have been obtained for special classes of topological spaces (cf., for instance, van Benthem and Bezhanishvili 2007; Bezhanishvili et al. 2003, 2004, or Gabelaia 2001). For this paper, the following theorem is fundamental:

Theorem 2.4 (Gabelaia 2003, Theorem 1.3.7, 1.3.8, pp. 16-17) ${ }^{2}$ Let WSC be the class of weakly scattered spaces. Then, the modal logic L(WSC) that corresponds to WSC is S4.1, i.e., the extension of S4 by the McKinsey axiom.

(MK)

$\square \diamond \mathrm{A} \Rightarrow \diamond \square \mathrm{A}$.

The topological translation of (MK) is

$(\mathrm{MK})_{\text {top }}$

$\operatorname{int}(\operatorname{cl}(\mathrm{A})) \subseteq \operatorname{cl}(\operatorname{int}(\mathrm{A}))$

\subsection{Examples and Counter-examples}

(i) $(\mathrm{X}, \mathrm{PX})$ is a topological space called the discrete topological space on $\mathrm{X}$. Clearly, (X, PX) is weakly scattered, since all points of $\mathrm{X}$ are isolated.

(ii) For any set $X$, the structure $(X,\{\varnothing, X\})$ is a topological space called the indiscrete topology on $X$. If $X$ has more than one element, $(X,\{\varnothing, X\})$ is not weakly scattered, since no point is isolated; rather, $\operatorname{int}(\{x\})=\varnothing$ for all $x \in X$. Any non-empty set $\mathrm{A} \subseteq \mathrm{X}$ is dense with respect to the indiscrete topology (X, $\{\varnothing, X\})$.

(iii) Let $X$ be the set $\{0,1\}$ of two elements and $O X=\{\varnothing,\{0\}, X\}$. The topological space $(\mathrm{X}, \mathrm{OX})$ is called the Sierpinski space. The only isolated point is point $\{0\}$, and clearly, $\operatorname{cl}(\{0\})=\{0,1\}$. Thus, the Sierpinski space is weakly scattered. Set $\{0\}$ of the Sierpinski space is dense, and set $\{1\}$ is closed and nowhere dense. It is not discrete, however.

(iv) The real line (R, OR) endowed with the standard Euclidean topology is not weakly scattered, it does not satisfy the McKinsey axiom $(\mathrm{MK})_{\text {top }}$ : Let $\mathbf{Q} \subseteq \mathbf{R}$ be the set of rational numbers. Then, $\operatorname{intcl}(\mathbf{Q})=\mathbf{R} \nsubseteq \subset \operatorname{cl}(\operatorname{int}(\mathbf{Q}))=\emptyset$. The Euclid-

\footnotetext{
${ }_{1}$ Recall that a topological space $(\mathrm{X}, \mathrm{OX})$ is Alexandroff if the arbitrary intersection of open sets is open, or, equivalently, if the arbitrary union of closed sets is closed.

2 Gabelaia's terminology is somewhat different. To ensure that Gabelaia's results are really equivalent to Theorem 2.4, the reader may consult Sect. 7 of this paper, particularly Lemmas 7.5 and 7.6. In most of the mathematical literature, Theorem 2.4 is considered "folklore" for which it is not necessary to give an exact source; see, e.g. Bezhanishvili et al. (2003).
} 
ean line ( $\mathbf{R}, \mathrm{OR})$ lacks any isolated points. The set $\mathbf{Q} \subseteq \mathbf{R}$ of rational numbers is dense in $\mathbf{R}$. Set $\mathbf{Z}$ of integers is nowhere dense in $\mathbf{R}$.

These examples and counter-examples show that the concept of weakly scattered spaces is consistent and non-trivial: There are topological spaces that satisfy the requirements for weakly scattered spaces, e.g., (X, PX) or the Sierpinsiki space ( $\{0$, $1\},\{\varnothing,\{0\}, X\})$, but not all topological spaces (X, OX) are weakly scattered.

Of course, being consistent and non-trivial is not a compelling argument for the usefulness of the concept of weakly scattered spaces. Therefore, in the next section, we will introduce several examples of interesting weakly scattered spaces that are naturally related to issues of vagueness. More precisely, following Rumfitt (2015) we will show that weakly scattered spaces provide a convenient topological framework for making precise Sainsbury's idea that vague concepts should be considered "boundaryless" concepts that are defined by reference to prototypes or paradigms (cf. Sainsbury 1990; Wright 1992).

\section{Weakly Scattered Spaces as Topological Models of Columnar Vagueness}

Following Bobzien, vagueness may be defined in a framework of a modal logic based on an operator $\mathbf{C}$ such that $\mathbf{C A}$ is to be interpreted as "It is clear that A", "It is definitely the case that A", or similarly. In the following, $\mathbf{C}$ is assumed to be an S4 operator (see Bobzien 2015). ${ }^{3}$ This means that the logic of $\mathbf{C}$ satisfies the following well-known conditions (cf. Dunn and Hardegree 2001):

Definition 3.1 The modal logic of the clearness operator $\mathbf{C}$ is defined as an extension of classical Boolean propositional logic by a unary sentential operator $\mathbf{C}$ such that for all well formed formulas $\mathrm{A}$ and $\mathrm{B}$, the following expression are valid:

\begin{tabular}{llc}
\hline (1) & $\mathbf{C}(\mathrm{A} \longrightarrow \mathrm{B}) \rightarrow(\mathrm{CA} \longrightarrow \mathrm{CB})$ & (Axiom K) \\
$(2)$ & $\mathrm{CA} \rightarrow \mathrm{A}$ & (Axiom T) \\
$(3)$ & $\mathrm{CA} \rightarrow \mathrm{CCA}$ & (Axiom 4) \\
(4) & If $-\mathrm{A}$, then $-\mathrm{CA}$. & (Rule of \\
& & Necessita- \\
& & tion).
\end{tabular}

With the aid of $\mathbf{C}$, operator $\mathbf{U}$ is defined as

\footnotetext{
3 This assumption is far from unanimously accepted. Rather, it is a quite controversial issue. For a variety of arguments in favour and against of this assumption see Bobzien (2015), Heck (1993), Williamson (1994) and Wright (2007). For a general survey see Zardini (2006). The topological perspective of this paper does not yield a direct argument in favour or against the thesis that a reasonable theory of vagueness has to be developed in the framework of S4. At least, it elucidates some of the consequences of this thesis in a particularly "intuitive" way (for those who consider the topological way of thinking as more intuitive than the set-theoretical one).
} 


\section{$\mathbf{U A}=$ NOT CA $\&$ NOT $(\mathbf{C}($ NOTA $)))$}

$\mathbf{U}$ is to be interpreted as unclearness in the sense that $\mathbf{U A}$ is to be read as "It is not clear that A, and it is not clear that not A". Abbreviating the $n$th iteration $\mathbf{U} \bullet \ldots \bullet \mathbf{U}$ of $\mathbf{U}$ by $\mathbf{U}^{\mathrm{n}}(\mathrm{n} \geq 1)$, Bobzien $(2015,2013)$ defines an account of vagueness as colum$n a r^{4}$ if and only if its unclearness operator $\mathrm{U}$ satisfies the equation

$$
\mathbf{U}^{\mathrm{n}} \mathrm{A}=\mathbf{U A}^{1} \text { for all } \mathrm{A} \text { and } \mathrm{n} \geq 1 .
$$

In general, (3.3) is not satisfied if the modal logic of clearness operator $\mathbf{C}$ is just logic S4 defined in Definition 3.1. ${ }^{5}$ Rather, Bobzien (2015, section II) shows that the validity of (3.3) requires a strengthening of S4, namely, S4.1, characterized as the extension of S4 by the so-called McKinsey axiom (MK) (cf. Theorem 2.4):

\section{$\mathbf{C N O T} \mathbf{C N O T} \mathrm{A} \Rightarrow$ NOT C NOT C A}

McKinsey and Tarski (1944) proved that modal system S4 is the logic of all topological spaces in the sense that a proposition is valid in S4 if and only if its topological interpretation is valid in all topological spaces. They thereby laid the foundation for the new discipline of "spatial logic", which would become a fruitful symbiosis of modal logic and topology. For a contemporary survey, see van Benthem and Bezhanishvili (2007). McKinsey and Tarski's result has been generalized in many ways, with a correspondence established between certain classes of topological spaces on the one hand and certain extensions S4.X of standard S4 logic on the other. For the purposes of this paper, the following special case is relevant: Let WSC denote the class of weakly scattered topological spaces (to be defined precisely in the next section). Then, the extension of S4 corresponding to this class of spaces is S4.1 (cf. van Benthem and Bezhanishvili 2007; Bezhanishvili et al. 2003, 2004; Gabelaia 2001).

In the following, we will exploit the correspondence between the S4.1 modal logic and the topology of weakly scattered spaces $(\mathrm{X}, \mathrm{OX})$ to elucidate the formal properties of columnar vagueness. Then, from a topological perspective, the clearness operator $\mathbf{C}$ corresponds to the topological operator int of the interior, and its operator $\mathbf{U}$ of unclearness corresponds to topological boundary operator bd defined by

$$
\operatorname{bd}(\mathrm{A}):=\operatorname{Cint}(\mathrm{A}) \& \operatorname{Cint}(\mathrm{CA})=\operatorname{cl}(\mathrm{A}) \cap \operatorname{cl}(\mathrm{CA})
$$

In terms of the topological concept of a boundary, the basic claim of the columnar account of vagueness reads

$$
\mathrm{bd}(\mathrm{bd}(\mathrm{A}))=\mathrm{bd}(\mathrm{A}) \quad \text { for all } \mathrm{A} \subseteq \mathrm{X}
$$

\footnotetext{
4 The "columnar higher-order vagueness" issue was first discussed in Bobzien (2010), and the term itself was introduced in Bobzien (2013).

5 In $\mathrm{S} 4$, the weaker equation $\mathrm{U}^{\mathrm{n}} \mathrm{A}=\mathrm{U}^{2} \mathrm{~A}$ can be proved for all $\mathrm{A}$ and $\mathrm{n} \geq 2$; see Sect. 7, Corollary 7.4.
} 
As is well known, (3.6) does not hold for all topological spaces (X, OX) and all $\mathrm{A} \subseteq \mathrm{X} .{ }^{6}$ Thus, the following definition is meaningful:

Definition 3.7 Let $(\mathrm{X}, \mathrm{OX})$ be a topological space. A subset $\mathrm{A} \subseteq \mathrm{X}$ is called a columnar set (with respect to the topology $\mathrm{OX}$ ) iff $\operatorname{bdbd}(\mathrm{A})=\mathrm{bd}(\mathrm{A})$.

Bobzien's result that $\mathrm{S} 4.1$ can be characterized as the modal logic of $\mathbf{C}$ for which corresponding uncertainty operator $\mathbf{U}$ satisfies $\mathbf{U}^{2}=\mathbf{U}$ is translated by Theorem 2.4 in the following theorem of topology:

Theorem 3.8 A topological space $(X, O X)$ is a weakly scattered space iff all subsets $A$ of $X$ are columnar; i.e., for all $A$, one has $b d(b d(A))=b d(A)$.

The fact that $\mathbf{Q}$ is not columnar in Euclidean space $(\mathbf{R}, \mathbf{O R})$ shows that familiar topological spaces such as the real line $(\mathbf{R}, \mathrm{OR})$ may differ considerably from weakly scattered spaces. Indeed, as will be shown in the following sections, weakly scattered spaces have some very peculiar properties.

Bobzien and Rumfitt (2019) sketch a proof that for logic S4.1, operators C and $\mathbf{U}$ are interdefinable. The topological translation of this fact asserts that for weakly scattered spaces (X, OX), interior operator int and boundary operator bd are interdefinable. This result can be strengthened. Already in 1927, Zarycki proved that for all topological spaces (X, OX), boundary operator bd and interior operator int are interdefinable (cf. Zarycki 1927). ${ }^{7}$ In logical terms, this amounts to the fact that for modal logic S4, operator $\square$ and contingency operator $\nabla$ are interdefinable, although the correspondence is more complex than that of $\mathbf{C}$ and $\mathbf{U}$ given for $\mathbf{S} 4.1$ by Bobzien and Rumfitt.

Moreover, in Sect. 6, we will show that for all topological spaces (X, OX), many subsets $A$ of $X$ are columnar; i.e., they satisfy $\overline{U A}=\mathbf{U}^{2} A$. This suggests that columnarity is a rather common phenomenon.

From now on, with the exception of Sect. 8, we will use topological terminology throughout the paper. That is, instead of using modal operators $\mathbf{C}$ and $\mathbf{U}$, we will use the corresponding topological operators int and bd. The only remaining reference to a logically motivated terminology will be that the expressions "columnar boundary" and "columnar set" are used to refer to a topological boundary operator bd that satisfies $b^{2}=b d$.

From the viewpoint of traditional topology, weakly scattered spaces are a rather elusive species. Familiar topological spaces such as Euclidean spaces and their derivatives are anything but weakly scattered. Thus, if one intends to use topological means for the explication of concepts such as vagueness and borderlineness, it is desirable to have a reservoir of "concrete" weakly scattered spaces that are "naturally related" to matters of vagueness. Here, the polar spaces of Rumfitt come to the

\footnotetext{
${ }^{6}$ The real numbers $\mathbf{R}$ endowed with the familiar Euclidean topology OR and subset $\mathbf{Q}$ of rational numbers of $\mathbf{R}$ provide an elementary counterexample since bd $(\mathbf{Q})=\mathbf{R}$ and bdbd $(\mathbf{Q})=\varnothing$ (see Sect. 7).

7 Another proof of this result may be found in Mormann (2013).
} 
rescue. It will be shown that polar spaces, recently introduced by Rumfitt (2015), provide a precise formal reconstruction of Sainsbury's idea that vague concepts such as colour concepts appear to be "boundaryless" concepts.

The concept of columnar boundaries makes sense not only for weakly scattered spaces but also for topological spaces in general. We will show that for all topological spaces (X, OX), many subsets are columnar. Moreover, for all topological spaces the set of columnar subsets has some nice structural properties, namely, it is a Boolean algebra.

\section{Weakly Scattered Spaces as a Framework for Prototypically Defined Concepts}

The aim of this section is to show that there are plenty of weakly scattered spaces that are relevant for issues of vagueness.

Our starting point is Sainsbury's influential idea that typical vague predicates such as "bald", "tall", "rich", and "red". are defined not by determining their boundaries but by referencing positive and negative paradigms or prototypes (cf. Sainsbury 1990). As Rumfitt put it:

Sainsbury invites us to consider a very familiar case: the colour spectrum, as displayed, for example, in an illustration in a book on colour. Looking carefully, we can discern no boundaries between the different colours: they stand out as clearly different, yet there are no sharp divisions. There are bands, but no bounds. This does nothing to impede the classificatory process: The spectrum is a paradigm of classification. (Sainsbury 1990, 258) The spectrum enables us to attach senses to colour terms not because it shows boundaries but because it displays colour paradigms or poles. ... [I] liken paradigms to gravitational poles, that is, massive bodies. If a small body is sufficiently close to a gravitational pole, it will be drawn towards it, rather as we are drawn to classify as red those objects that are sufficiently close in colour to a paradigm, or pole, of red. (Rumfitt 2015, 236)

From an extensional, set-theoretical point of view, "red" and "non-red" are logically and conceptually on par: One is just the negation of the other. The prototypical approach defends a quite different point of view: Even if there is a paradigm of "red", there is no paradigm of "non-red". Rather, any other colour will serve as a paradigm of how not to be red but only by virtue of its positive classification as another colour (cf. Sainsbury 1990, 258). ${ }^{8}$ As Rumfitt shows, the distinction of a set $\mathrm{P}$ of prototypical elements in a domain $\mathrm{X}$ naturally endows it with a topological structure OX. Following Rumfitt, topological structures obtained in this way are

\footnotetext{
${ }^{8}$ In some sense, the prototypical classification of colours works in a similar way as the characterization of implicitly defined mathematical concepts works. A system of geometrical concepts such as "points", "lines", and "planes" is grasped as a whole. Thus, "point" is an important geometrical concept defined in relation to other geometrical concepts, but "non-point" is clearly not.
} 
called polar topological spaces. As will be shown in this paper, polar topological spaces can be characterized as weakly scattered spaces that we already met in the topological translation of Bobzien's account of columnar vagueness.

The necessary ingredients for constructing a polar topology for a domain may be succinctly described as follows: Assume that there is given a set $\mathrm{X}$ of objects to be classified and a subset $\mathrm{P}$ of $\mathrm{X}$ to be considered as a set of distinguished elements that are "paradigmatic" or "prototypical" objects of X. In the terminology of Rumfitt (2015), they are called poles. Set P of poles is used to classify the objects of $\mathrm{X}$ as follows:

Definition 4.1 (Pole Distribution for $X$ ) Let $\mathrm{X}$ be a non-empty set, $\mathrm{P}$ be a nonempty subset of $\mathrm{X}$, and $2^{\mathrm{P}}$ be the power set of $\mathrm{A}$. A function $\mathrm{X}-\mathrm{m} \longrightarrow 2^{\mathrm{P}}$ is a pole distribution iff it satisfies the following requirements:

A pole distribution is denoted by $(\mathrm{X}, \mathrm{m}, \mathrm{P})$.

For colour experiences, the working of a pole distribution can be described as follows: Each shade of colour may be assumed to be in the "domain of influence" of at least one prototype. This means that if a is a shade of blue sufficiently similar to B, then $m(a)=\{B\}$. If, however, a competent non-colour-blind observer feels unable to say whether $a$ is red or blue, then $m(a)=\{R, B\}$. It seems plausible to assume that for all prototypical experiences $\mathrm{p} \in \mathrm{P}$, a polar distribution $\mathrm{m}$ satisfies $\mathrm{m}(\mathrm{p})=\{\mathrm{p}\}$; i.e., every prototypical experience $\mathrm{p}$ is only in the sphere of influence of itself. Epistemically, this means that different poles are easily distinguishable, i.e., epistemologically clearly different.

Rumfitt observed that a prototype distribution (X, m, P) defines a topology on $\mathrm{X}$ (cf. Rumfitt 2015, 243, Fn. 15):

Proposition 4.2 A pole distribution $(X, m, P)$ defines a topology $(X, O X)$ by an interior kernel operator $P X \longrightarrow$ int $\longrightarrow P X$

$$
\mathrm{y} \in \operatorname{int}(\mathrm{A}):=\mathrm{y} \in \mathrm{A} \text { and } \forall \mathrm{p} \in \mathrm{P}(\mathrm{p} \in \mathrm{m}(\mathrm{y}) \Rightarrow \mathrm{p} \in \mathrm{A}) \quad(\mathrm{A} \subseteq \mathrm{X})
$$

The topological space $(\mathrm{X}, \mathrm{OX})$ is called a polar topological space. Subset $\mathrm{P} \subseteq \mathrm{X}$ is called the set of poles.

Proof To check that int satisfies Kuratowski axioms (1)*-(4)* of Definition 2.1, see Rumfitt (2015, 243-244).

Proposition 4.3 Let $(X, O X)$ be the topological space defined by a pole distribution $(X, m, P)$. For all $x \in X$, the smallest open set of the polar topology $O X$ that contains $x$ is set $V(x):=\{x\} \cup m(x)$. For elements $p \in P$ and $x \in X-P$, one calculates 


\begin{tabular}{lll}
\hline $\operatorname{int}(p)=\{p\}$, & $\operatorname{int}(x)=\emptyset$, & $V(x)=\{x\} \cup m(x)$ \\
$\operatorname{cl}(x)=\{x\}$, & $\operatorname{cl}(p)=\{x ; p \in m(x)\}$, & $\operatorname{int}(\operatorname{cl}(p))=\{x ;\{p\}=m(x)\}$
\end{tabular}

Proof Let us prove $\mathrm{V}(\mathrm{x})=\{\mathrm{x}\} \cup \mathrm{m}(\mathrm{x})$. According to the definition of the operator int, one has $y \in \operatorname{int}(\{x\} \cup m(x)) \Leftrightarrow y \in\{x\} \cup m(x) \& \forall p(p \in m(y) \Rightarrow p \in\{x\} \cup m(x))$. Clearly, every element in $\{x\} \cup m(x)$ satisfies this condition. On the other hand, any smaller set properly containing $\{x\} \cup \mathrm{m}(\mathrm{x})$ clearly does not satisfy the condition. For two different elements, $x$ and $y \mathrm{~V}(\mathrm{x})$ and $\mathrm{V}(\mathrm{y})$ are different. Hence, $\mathrm{X}$ is a $\mathrm{T}_{0}$ space. Thus, sets $\{\{x\} \cup \mathrm{m}(\mathrm{x}), \mathrm{x} \in \mathrm{X}\}$ form a unique minimal base for polar topology $\mathrm{OX}$ on $\mathrm{X} .{ }^{9}$

From Proposition 4.3, one immediately obtains a pleasing corollary that relates Rumfitt's account of polar spaces with Bobzien's account of columnar vagueness:

Corollary 4.4 A polar topological space $(X, O X)$ is weakly scattered. More precisely, the set of isolated points ISO $(X)$ of $X$ is just the set $P$ of poles. This set is dense in $X$, i.e., $\operatorname{cl}(P)=\operatorname{cl}(I S O(X)=X$.

Proof First, we show that the singletons $\{p\}$ of poles $p \in P$ are open in the polar topology, i.e., $\{p\} \in O X$. Then, it is proved that $\operatorname{cl}(P)=X$. Let $q \in P$. By Proposition 4.3 of int for $\{q\}$, one has $y \in \operatorname{int}(q)$ iff $y \in\{q\}$ and $\forall p(p \in P$ and $\mathrm{p} \in \mathrm{m}(\mathrm{y}) \Rightarrow \mathrm{p} \in \mathrm{A})$. By definition of a pole distribution $\mathrm{m}$, for a pole $\mathrm{q}$, there is only one pole in $\mathrm{m}(\mathrm{q})$, namely, $\mathrm{q}$ itself. Hence, $\{\mathrm{q}\}$ is open for $\mathrm{q} \in \mathrm{P}$. Only slightly more difficult is the calculation of $\operatorname{cl}(q)$ : By definition, closure operator cl of $(X, O X)$ is given by (cf. Rumfitt 2015, p. 244) $x \in \operatorname{cl}(A):=x \in A$ or $\exists p \in P(p \in m(x)$ and $p \in A)$. For $A=\{q\}, q \in P$, this yields $\operatorname{cl}(q):=\{x ; q \in m(x)\}$. To show that set $P$ of poles is just the set $\operatorname{ISO}(X)$ of the isolated elements of $X$ and is dense in $X$, one first observes that $X$ is Alexandroff; therefore, one has $\mathrm{cl}(\mathrm{P})=\cup_{\mathrm{p} \in \mathrm{P}} \mathrm{cl}(\mathrm{p})$. Now, by definition of $\mathrm{m}$ for all $\mathrm{x}$, set $\mathrm{m}(\mathrm{x})$ is not empty. Hence, every $\mathrm{x}$ is an element of at least one set $\mathrm{cl}(\mathrm{p})$. Therefore, $\mathrm{cl}(\mathrm{P})$ is dense in $\mathrm{X}$; i.e., $\mathrm{X}$ is weakly scattered.

Corollary 4.5 Let $(X, O X)$ be the polar space defined by the distribution $(X, m, P)$. Denote the Boolean algebra of regular open subsets by $O * X$. Then, $O * X$ is isomorphic to $2^{P}$ of $P$ by the map $2^{P}-$ intcl $\longrightarrow O * X$ defined for $Q \in 2^{P}$ by

$$
\operatorname{intcl}(Q):=\operatorname{intcl}(\cup\{\operatorname{intcl}(\mathrm{q}) ; \mathrm{q} \in \mathrm{Q}\} . \diamond
$$

It is easily proved that the polar topology $(X, O X)$ of the colour circle is not trivial, i.e., $O X \neq\{\emptyset, X\}, P X$. More interesting is the fact that it does not satisfy the Brouwer axiom $(B)$ and the converse $M K^{*}$ of the McKinsey axiom:

\footnotetext{
${ }^{9}$ The existence of the minimal basis V(x) renders (X, OX) an Alexandroff space (cf. Alexandroff 1937).
} 
Proposition 4.6 Let $(X, O X)$ be the colour circle endowed with the polar topology. Then, neither Brouwer's axiom B nor the converse MK* of McKinsey's axiom is valid $^{10}$ :

Brouwer's axiom B

Converse McKinsey axiom $(M K)^{*}$
$C A \subseteq \operatorname{int}(C(\operatorname{int}(C A))$

$\operatorname{cl}(\operatorname{int}(A)) \subseteq \operatorname{int}(\operatorname{cl}(A))$

Proof Let $(\mathrm{X}, \mathrm{OX})$ be the polar topology on $\mathrm{X}$ defined by a polar distribution $(X, m, P)$. We may assume that for $p \in P$, one has $\operatorname{intcl}(p)) \neq\{p\}$. That is, $\{x$; $\mathrm{m}(\mathrm{x})=\{\mathrm{p}\}\} \neq\{\mathrm{p}\}$. Take $\mathrm{A}=\mathrm{X}-\{\mathrm{p}\}$. Then, Brouwer's axiom (B) requires that $\{\mathrm{p}\}$ $\subseteq \operatorname{int}(C(\operatorname{int}(C(X-\{p\}))=\operatorname{int}(X-\{p\})$. Clearly, however, $\{p\} \not \subset \operatorname{int}(X-\{p\})$. Hence, the polar space $(\mathrm{X}, \mathrm{OX})$ does not satisfy $(\mathrm{B})$.

The converse (MK)* of the McKinsey axiom (MK) is disproved by considering $A=\{p\}, p \in P$. Then, one obtains $\operatorname{cl}(\operatorname{int}(p))=\{x ; p \in m(x)\}$ and $\operatorname{int}(\operatorname{cl}(p))=\{x$; $\{\mathrm{p}\}=\mathrm{m}(\mathrm{x})\}$. Clearly, $\{\mathrm{x} ; \mathrm{p} \in \mathrm{m}(\mathrm{x})\} \not \subset \operatorname{intcl}(\mathrm{p}))=\{\mathrm{x} ;\{\mathrm{p}\}=\mathrm{m}(\mathrm{x})\}$. Thus, $(\mathrm{MK})^{*}$ does not hold.

Proposition 4.3 offers a complete description of the topology of polar spaces (X, $\mathrm{OX}$ ). Let us check that intuitively satisfying results are obtained for the paradigmatic example of the colour spectrum. More precisely, let $\mathrm{S}$ be the set of colour experiences and $\mathrm{P} \subseteq \mathrm{S}$ be a finite subset of paradigmatic colour experiences such as "red" (r), "orange" (o), "blue" (b), and "yellow" (y). Let (S, m, P) be an appropriate polar distribution $\mathrm{S}-\mathrm{m}-2^{\mathrm{P}}$. According to Rumfitt, the extension of a basic colour concept such as "red" is given by the regular open subset of S intcl(r) generated by $\{r\}$. One calculates, as it should be, $\operatorname{int}(\operatorname{cl}(r))=\{x ; m(x)=\{r\}\}$. In other words, the extension of the concept "red" is given by the set of all colour experiences that are related to only one prototype, namely, r. Experiences related to two paradigmatic colour experiences, e.g., "orange" and "red", are described as those $\mathrm{x} \in \mathrm{S}$ with $\mathrm{m}(\mathrm{x})=\{\mathrm{r}, \mathrm{o}\} .{ }^{11}$ Proposition 4.3 also offers a compelling example that open but not regular open subsets cannot be considered to represent "natural" concepts: Given intcl(r), assume $G \subseteq$ intcl(r)) a set of non-prototypical shades of red. By Proposition 4.3, set G is closed. Hence, set (intcl(r)) - G) is open but, of course, not regular open. Obviously, (intcl(r)) $-\mathrm{G})$ can hardly be considered the extension of a well-behaved colour predicate, since any "arbitrary" set G of shades is missing. This

\footnotetext{
10 The axioms (MK)* and (B) have a certain relevance for epistemological matters: (MK)* is characteristic for modal system S4.2 corresponding to the class of extremally disconnected spaces that have proven useful for modeling the concept of knowledge (cf. Baltag et al. 2018). Brouwer's axiom (B) is an axiom of Williamson's logic of clarity (cf. Williamson 1994, 1999) based on modal operator $\mathbf{C}^{*}$; see Sect. 8. This emphasizes the necessity of strictly distinguishing operators $\mathbf{C}$ and $\mathbf{C}^{*}$, although their informal characterization in English may seem rather similar.

${ }^{11}$ For the linear colour spectrum, all colour experiences can be influenced by at most two prototypical experiences, but of course, there may be polar spaces for which mixed non-paradigmatic experiences of three or more prototypical experiences exist.
} 
confirms Rumfitt's conjecture that the extension of any colour predicate is to be a regular open set (cf. Rumfitt 2015, p. 244/245). ${ }^{12}$

Rumfitt discusses his topological reconstruction of Sainsbury's idea by linear colour spectrum $\mathrm{S}$ whose poles are linearly ordered from red on the left end and blue on the right side of the spectrum (cf. Rumfitt 2015, 235ff). As is well known, this system is not the only system that can be used to order colour experiences. For instance, instead of a linear structure, one may use a colour circle in which colours are circularly ordered and the ends of the linear spectrum are identified. The resulting topological space is again a polar space. As Rumfitt rightly points out, the construction of a polar topological space can be carried out for any vague concept whatsoever provided that it is defined by prototypes. A fortiori, the linear colour spectrum as well as the colour circle yield (different) polar topological spaces. For our considerations this difference is not relevant.

In sum, for all domains X endowed with a polar distribution (X, m, P), Rumfitt's construction can be carried out. ${ }^{13}$ Vague concepts of $\mathrm{X}$ have regular open extensions

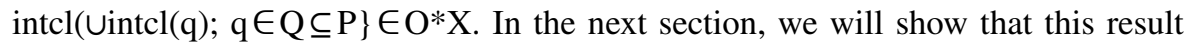
can be used to obtain a solution to the Sorites paradox based on classical Boolean logic and (not-so-classical) regular open semantics.

Clearly, polar topological spaces differ considerably from more familiar topological spaces, such as Euclidean spaces. For instance, in contrast to Euclidean spaces (non-trivial), polar topologies do not satisfy separation axioms $\mathrm{T}_{2}$ and $\mathrm{T}_{1}$, and they have isolated points. These differences should be considered an advantage. Consider the colour spectrum or the colour circle. Usually, the underlying sets of these spaces are thought to be endowed with a (Euclidean) metric. This structure produces many representational artefacts that lack any possible empirical meaning. For instance, what does it mean that certain colour experiences $x, y, z$, and $w$ are such that the distance between $\mathrm{x}$ and $\mathrm{y}$ is the same as the distance between $\mathrm{z}$ and $\mathrm{w}$ ? It seems difficult, to say the least, to interpret such an assertion (or its negation) as empirically meaningful. In contrast, assuming that the colour circle is endowed only with a much more modest polar topology, such empirically unfounded assertions cannot even be formulated.

\footnotetext{
12 This claim has to be understood with appropriate qualifications, of course. Otherwise it is obviously wrong. Clearly, colour concepts like "scarlet", "maroon", "turquoise", or "olive" cannot be expressed in a language that is based on a simple pole distribution $(\mathrm{X}, \mathrm{m}, \mathrm{P})$ possessing only the basic terms "red", "green", etc. Rather, what is needed to express "scarlet" is a pole distribution (X, $\left.\mathrm{m}^{\prime}, \mathrm{P}^{\prime}\right)$ such that either "scarlet" has its own pole $\mathrm{p}^{\prime} \in \mathrm{P}^{\prime}$, or the extension of "scarlet" is the (connected) sum of two (or more) regular open subsets of $\left(\mathrm{X}, \mathrm{O}^{\prime} \mathrm{X}\right), \mathrm{O}^{\prime} \mathrm{X}$ defined by $\left(\mathrm{X}, \mathrm{m}^{\prime}, \mathrm{P}^{\prime}\right)$.

${ }^{13}$ Two special cases of polar distributions may be particularly mentioned. Clearly, if $\mathrm{P}=\{\mathrm{p}\} \subseteq \mathrm{X}$, every distribution $(X, m, P)$ is trivial, i.e., $m(x)=\{p\}$ for $x \in X$. More interesting is the case $P=\left\{p, p^{\prime}\right\}$. In this case, $\mathrm{p}$ and p' may be interpreted as prototypes of opposite concepts, e.g. "rich" and "poor", "silly" and "intelligent" etc. In many paradigmatic cases of concepts for which a kind of Sorites makes sense, however, opposites have to be introduced more or less artificially. For instance, if $\mathrm{X}$ is the class of men, then the opposite of "bald" is something like "having a scalp with at least 100.000 hairs". This, however, is not an insuperable obstacle to apply the apparatus of polar distributions also to these cases.
} 


\section{Rumfitt's Solution to the Sorites Paradox in the Framework of Classical Logic and Regular Open Semantics}

Given a topological space $(\mathrm{X}, \mathrm{OX})$, set $\mathrm{O} * \mathrm{X}$ of regular open subsets of $\mathrm{X}$ is defined as the subset of elements $\mathrm{A}$ of $\mathrm{OX}$ that satisfy $\mathrm{A}=\operatorname{int}(\mathrm{cl}(\mathrm{A}))$. As is well known (cf. Tarski 1938, p. 448) $\mathrm{O} * \mathrm{X}$ can be endowed with the structure of a complete Boolean algebra with respect to operations $\wedge$ ("and"), $\vee$ ("or") and $\neg$ ("not") defined as follows:
(i) $\mathrm{A} \wedge \mathrm{B}=\mathrm{A} \cap \mathrm{B}$,
(ii) $\mathrm{A} \vee \mathrm{B}:=\operatorname{intcl}(\mathrm{A} \cup \mathrm{B})$,
(iii) $\neg \mathrm{A}:=\operatorname{int}(\mathrm{CA})$

Using (5.1), $\mathrm{O} * \mathrm{X}$ can be used to define an adequate interpretation for the calculus of classical propositional logic as follows:

Definition 5.2 ${ }^{14}$ Let L be a propositional language with propositional variables, $\mathrm{a}, \mathrm{b}, \ldots$, and connectives $\wedge, \vee, \longrightarrow$ and $\neg$ to be interpreted as "and", "or" "if ... then", and "not". Let $(\mathrm{X}, \mathrm{OX})$ be a topological space and $\mathrm{O} * \mathrm{X}$ be the Boolean algebra of regular open sets. A regular open interpretation of $L$ in $O * X$ is a map $\mathrm{L}-\mathrm{r}->\mathrm{O} * \mathrm{X}$, for which $\mathrm{r}(\mathrm{a}) \in \mathrm{O} * \mathrm{X}$, such that

\begin{tabular}{llll}
\hline (1) & $\mathrm{r}(\mathrm{a} \wedge \mathrm{b}):=\mathrm{r}(\mathrm{a}) \cap \mathrm{r}(\mathrm{b})$. & $(2)$ & $\mathrm{r}(\mathrm{a} \vee \mathrm{b}):=\operatorname{intcl}(\mathrm{r}(\mathrm{a}) \cup \mathrm{r}(\mathrm{b}))$, \\
(3) & $\mathrm{r}(\mathrm{a} \longrightarrow \mathrm{b}):=\operatorname{int}(\mathrm{Cr}(\mathrm{a}) \cup \mathrm{r}(\mathrm{b}))$. & $(4)$ & $\mathrm{r}(\neg \mathrm{a}):=\operatorname{Ccl}(\mathrm{r}(\mathrm{a}))$.
\end{tabular}

A regular open interpretation boils down to a familiar set-theoretical interpretation if $(\mathrm{X}, \mathrm{OX})=(\mathrm{X}, \mathrm{PX})$.

The main advantage of a regular open interpretation resides in the interpretation of disjunctions of regular open concepts that Rumfitt uses to defuse the notorious inductive argument of the Sorites paradox in an elegant way. Before we discuss this in detail, let us consider the following toy example:

Example 5.3 Let $\mathrm{X}=\{\mathrm{N}, 1,2, \ldots, 100, \mathrm{~S}\}$ and $(\mathrm{X}, \mathrm{m}, \mathrm{P})$ be the polar distribution defined by $m(N)=\{N\}, m(S)=\{S\}$, and $m(i)=\{S, N\}$ for $1 \leq i \leq 100$. Let $(X, O X)$ be the resulting polar topological space with interior operator int and closure operator cl. By Proposition 4.3, one calculates

$$
\begin{aligned}
& \operatorname{cl}(N)=\{1,2, \ldots, 100, N\}, \operatorname{intcl}(N)=\{N\} \\
& \operatorname{cl}(S)=\{1,2, \ldots, 100, S\}, \operatorname{intcl}(S)=\{S\}
\end{aligned}
$$

For the regular open disjunction of the regular open concepts intcl(N) and intcl(S), one calculates

\footnotetext{
14 This definition can be found already in Tarski (1938, Definition 4.1, p. 434 with comments on p. 448)).
} 


$$
\langle\mathrm{N}\rangle \vee\langle\mathrm{S}\rangle=\operatorname{intcl}(\{\mathrm{N}, \mathrm{S}\})=\{\mathrm{N}, 1, \ldots, 100, \mathrm{~S}\}=\mathrm{X} .
$$

That is, all $i \in X$ satisfy disjunction $\langle N\rangle \vee\langle S\rangle$, but none satisfies either $\langle N\rangle$ or $\langle S\rangle$. In other words, satisfaction does not distribute over the regular open disjunction $\mathrm{V}$. Expressed in terms of truth, for all $x \in X$, the proposition " $\mathrm{x}$ is $\langle\mathrm{N}\rangle \vee\langle\mathrm{S}\rangle$ " is true, but for most $\mathrm{x} \in \mathrm{X}$, the propositions " $\mathrm{x}$ is $\langle\mathrm{N}\rangle$ " and " $\mathrm{x}$ is $\langle\mathrm{S}\rangle$ " are false.

Based on a well-known argument of Wright (2007), Rumfitt shows that the Sorites argument leads to a paradox thesis of the existence of sharp cutoff points of a Sorites series. Starting with the following setting, the details are as follows:

Let us suppose, then, that we have a sequence of one hundred transparent tubes of paint, $a_{1}, \ldots, a_{100}$, with the following properties: tube $a_{1}$ is clearly red; tube $\mathrm{a}_{100}$ is clearly orange and hence clearly not red; but for each $\mathrm{n}$, tube $\mathrm{a}_{\mathrm{n}+1}$ is only marginally more orange (and hence only marginally less red) than its predecessor an. Indeed, let us suppose that an observer with good eyesight, when viewing any pair of adjacent tubes $a_{n}$ and $a_{n+1}$ together in white light but without comparing them with other tubes, is unable to perceive any difference in colour between them. That is, we suppose that any two adjacent members of the sequence are indiscriminable in colour. In such a case, the claim that nowhere in the sequence is a red tube immediately followed by a non-red tube seems highly plausible. (Rumfitt 2015, 220)

Faithfully following Wright's original argumentation, Rumfitt shows that using classical Boolean logic, this setting of adjacent pairwise indistinguishable transparent tubes leads to the following "unpalatable disjunction":

$$
\left(\mathrm{Ra}_{1} \& \neg \mathrm{Ra}_{2}\right) \vee\left(\mathrm{Ra}_{2} \& \neg \mathrm{Ra}_{3}\right) \vee \ldots \vee\left(\mathrm{Ra}_{99} \& \neg \mathrm{Ra}_{100}\right) \quad\left(\mathrm{Ra}_{\mathrm{i}}=\mathrm{a}_{\mathrm{i}} \text { is red }\right)
$$

This disjunction seems "unpalatable" since it apparently asserts that there is a tube $a_{i}$ that is red and adjacent to a tube $a_{i+1}$ that is indistinguishable in colour from $\mathrm{a}_{\mathrm{i}}$ but is not red. This would amount to admitting the existence of a sharp cutoff of the vague concept $R$, which seems absurd. This absurd assertion can be avoided by using regular open semantics of regular open predicates in the following way.

First, one should observe that for a given polar space (X, OX) defined by a polar distribution $(\mathrm{X}, \mathrm{m}, \mathrm{P})$, the Cartesian product $\mathrm{X}^{100}:=\mathrm{X} \times \ldots(100$ times $) \ldots \times \mathrm{X}$ can be considered a polar space whose polar structure is defined by $(\mathrm{X} \times \cdots \times \mathrm{X}, \mathrm{m} \times \cdots \times \mathrm{m}$, $\mathrm{P} \times \cdots \times \mathrm{P})$. Then, (5.4) can be conceived as a complex proposition about element $\mathrm{a}=\left\langle\mathrm{a}_{1}, \ldots, \mathrm{a}_{100\rangle} \in\right.$ of $\mathrm{X}^{100}$ by interpreting propositions $\mathrm{Ra}_{\mathrm{i}}$ as asserting that the $i$ th component of sequence a is red. Indeed, the predicate "the $i$ th component of a sequence $\left\langle\mathrm{x}_{1} \ldots, \mathrm{x}_{100}\right\rangle$ is red" is easily shown to be regular open in $\left(\mathrm{X}^{100}, \mathrm{OX}^{100}\right)$. In sum, (5.4) can be interpreted as a disjunction of regular open predicates of Cartesian product $\mathrm{X}^{100}$. As Rumfitt points out

When understood in that way, [(5.4)] is entirely innocuous. All it says is that when classifying entire sequences of coloured objects, whose members are arranged in order of gradually decreasing redness from something clearly red to something clearly not red, either the second or the third or... or the 100th 
object will be the first object not classified as red. That claim is obviously true. It does not, however, entail the existence of a sharp boundary to the concept red, which is a mode of classifying a single object in respect of its colour. (Rumfitt 2015, p. 253)

\section{Polar Spaces and Conceptual Spaces}

Polar spaces do not only arise as by-products in the topological reconstruction of prototypically defined vague concepts. A fruitful source for polar spaces can be found in cognitive psychology and related disciplines, in which conceptual spaces have played a crucial role since they were introduced some 20 years ago by Gärdenfors (see Gärdenfors 2000, 2014; Gärdenfors and Zenker 2015).

In its simplest version, a conceptual space is given by a metrical space $(S, d)$ endowed with a finite set $\mathrm{P} \subseteq \mathrm{S}$ of prototypes. The elements of $\mathrm{S}$ represent objects, experiences, or processes of a certain kind, for instance, colours, tastes, and sounds. Elements $\mathrm{x}$ and $\mathrm{y}$ of $\mathrm{S}$ become more similar to each other as their distance decreases. In the following, such a structure is denoted by $(\mathrm{S}, \mathrm{P}, \mathrm{d})$. Prototypes $\mathrm{P}$ serve as "centres of gravity" much in the same way that they do in the Sainsbury/Rumfitt approach of polar spaces. Conceptual spaces and polar spaces have in common that they describe concepts "more geometrico", namely, as appropriate regions of (topologically or geometrically structured) spaces. Thus, both accounts may be seen as versions of a spatial logic.

More precisely, conceptual spaces $(\mathrm{S}, \mathrm{P}, \mathrm{d})$ naturally give rise to polar spaces $(\mathrm{S}$, $\mathrm{P}, \mathrm{m}_{\mathrm{d}}$ ) as follows:

Proposition 6.1 Let $(S, P, d)$ be a conceptual space. For $x \in S$, define $P(x):=\{d(x$, $p) ; p \in P\}$. Since $P$ is finite, for all $P(x)$, its subset $M(x)$ of minimal elements is not empty. Moreover, for $p \in P$, one has $M(p)=\{p\}$. Then, a pole distribution $(S, m, P)$ with its polar space $(S, O S)$ is defined by $S \longrightarrow m \rightarrow 2^{P}$ and $m(x):=M(x)$.

Conceptual spaces $(\mathrm{S}, \mathrm{P}, \mathrm{d})$ and polar spaces $(\mathrm{X}, \mathrm{m}, \mathrm{P})$ differ in the kind of structure that is used for defining concepts as more geometrico. If $\mathrm{S}$ is assumed to be equipped with a Euclidean metric, categorization by prototypes results in a so-called Voronoi tessellation of conceptual space S. A characteristic feature of a Voronoi tessellation of a conceptual space is that the space is partitioned into finitely many convex regions. This led Gärdenfors to put forward "criterion P" as a central idea of his approach, in which "good", i.e., natural, concepts are geometrically singled out as convex regions (cf. Gärdenfors 2000, p.92/93):

6.2 Criterion P Let $(S, P, d)$ be a conceptual space $S$ endowed with a Euclidean metrical structure d. Then, a natural property of $(S, P, d)$ is a convex region of $S$.

The problem with "criterion P" is that Euclidean spaces possess a profusion of convex regions that, according to (6.2), must be considered natural concepts. 
Therefore, a profusion of infinitely many allegedly natural properties is generated. This lacks plausibility. As is easily seen, the polar approach does not suffer from this unwanted profusion of "natural" concepts. Moving from the conceptual space (S, P, d) to the polar distribution $\left(\mathrm{S}, \mathrm{m}_{\mathrm{d}}, \mathrm{P}\right)$, one ends up in polar space $(\mathrm{S}, \mathrm{OS})$ such that the atoms of $\mathrm{O} * \mathrm{~S}$ are just finitely many regular open cells of the Voronoi tessellation of $(\mathrm{S}, \mathrm{P}, \mathrm{d})$. In other words, the austere topological structure defined by $\left(\mathrm{S}, \mathrm{m}_{\mathrm{d}}, \mathrm{P}\right)$ suffices to generate the really important part of the categorizing tessellation of the conceptual space S, namely, the regular open cells of the tessellation. This means that the structural austerity of polar spaces really helps reduce problems caused by representational artefacts. Relying on $\left(\mathrm{S}, \mathrm{m}_{\mathrm{d}}, \mathrm{P}\right)$ instead of $(\mathrm{S}, \mathrm{P}, \mathrm{d})$ is a convenient way to avoid an infinite profusion of allegedly natural predicates. On the other hand, the framework of polar spaces is still sufficiently complex to draw important distinctions: For instance, polar structures suffice to distinguish topologically between the colour circle and the linear colour spectrum.

\section{The Boolean Algebra of Columnar Sets of a Topological Space}

In this section, we show that every topological space $(\mathrm{X}, \mathrm{OX})$ comes with a large class of columnar subsets $A \subseteq X$, i.e., satisfy $b d(b d(A))=b d(A)$. Moreover, the class of these subsets has the structure of a Boolean algebra (with respect to the set-theoretical operations inherited from set $\mathrm{X}$ ).

Definition 7.1 A set $A \subseteq X$ of a topological space (X, OX) is called a McKinsey set of $(X, O X)$ if and only if $b d(b d(A))=b d(A)$. The set of McKinsey sets of $(X, O X)$ is denoted by MKX.

If $(X, O X)$ is weakly scattered, all subsets of $X$ are columnar sets, i.e., $M K X=P X$. In general, however, this is not the case, as is shown by the following elementary example:

Example 7.2 Let (R, OR) be the real line with the standard Euclidean topology. Boundary $\operatorname{bd}(\mathbf{Q})$ of set $\mathbf{Q}$ of rational numbers is not columnar, i.e., $\operatorname{bd}(\mathbf{Q}) \neq \operatorname{bd}(\operatorname{bd}(\mathbf{Q}))$.

Proof As is easily calculated, $\mathbf{Q}$ and its complement $\mathbf{C} \mathbf{Q}$ are dense in $\mathbf{R}$. Hence, $\operatorname{bd}(\mathbf{Q})=\mathbf{R}$ and $\operatorname{bd}(\operatorname{bd}(\mathbf{Q}))=\operatorname{bd}(\operatorname{bd}(\operatorname{bd}(\mathbf{Q})))=\varnothing$.

Clearly, for all topological spaces (X, OX), one has MKX $\subseteq$ PX. Weakly scattered spaces $(\mathrm{X}, \mathrm{OX})$ are characterized as spaces for which MKX $=\mathrm{PX}$. In the rest of this section, we show that for all topological spaces, MKX has a very nice algebraic structure; namely, MKX is a Boolean algebra with respect to the set-theoretical operations $\cap, \cup$, and $C$. For the proof, we need some lemmas.

Lemma 7.3 Let (X, OX) be a topological space. Then, $O X \cup C X \subseteq M K X$; i.e., if $A$ is an open set or a closed set, then $b d(A)=b d(b d(A))$. 
Proof Assume A to be open. By definition, $b d(A)=\operatorname{cl}(A) \cap \operatorname{cl}(C A)=\operatorname{cl}(A) \cap C A$, since $A$ is open. For bd(bd(A)), one obtains $b d(b d(A))=b d((\operatorname{cl}(A) \cap C A))=(\operatorname{cl}(A) \cap$ $\mathrm{CA}) \cap \operatorname{cl}(\mathrm{C}(\mathrm{cl}(\mathrm{A}) \cap))$. Obviously, $\operatorname{cl}(\mathrm{C}(\mathrm{cl}(\mathrm{A}) \cap \mathrm{CA})=\operatorname{cl}(\mathrm{C}(\mathrm{cl}(\mathrm{A}) \cup \mathrm{CA}))=\operatorname{cl}(\mathrm{C}(\mathrm{cl}(\mathrm{A}))$ $U))=\operatorname{cl}(X)=X$. Hence, $\operatorname{bd}(\operatorname{bd}(A))=\operatorname{bd}(A)$.

The boundary of a closed set B equals the boundary of its open complement CB. Thus, the boundaries of open sets and those of closed sets are columnar.

Corollary 7.4 For all topological spaces $(X, O X)$, all $A \subseteq X$ are stably columnar, i.e., $b d(b d(b d(A)))=b d(b d(A))$.

Proof By definition, bd(A) is closed. Applying Proposition 4.3 to bd(A), one immediately obtains $b d(b d(b d(A)))=b d(b d(A))$.

Thus, in the general case, the column of higher-order boundaries $\left\{b^{n}(A)\right\}_{n \in N}$ of a set A starts with a "pedestal" bd(A) on which equal layers $b^{2}(A), b d^{3}(A), \ldots$ ad infinitum. ${ }^{15}$

Lemma 7.5 For all $(X, O X)$, one has $b d(a)=b d(b d(a))$ iff int $(b d(a))=\emptyset$.

Proof Assume bdbd(a)=bd(a). Then, calculate

$$
\begin{aligned}
\operatorname{bd}(a)=\operatorname{bd}(b d(a)) & \Leftrightarrow \operatorname{bd}(a)=\operatorname{bd}(a)) \cap \operatorname{cl}(\operatorname{Cbd}(a)) \Leftrightarrow \operatorname{bd}(a)=b d(a) \cap \operatorname{cl}(\operatorname{Cbd}(a)) \\
& \Leftrightarrow \operatorname{bd}(a) \subseteq \operatorname{cl}(\operatorname{Cbd}(a)) \Leftrightarrow \operatorname{bd}(a) \subseteq \operatorname{Cint}(b d(a)) \\
& \Leftrightarrow \operatorname{int}(b d(a)) \cap \operatorname{bd}(a)=\emptyset \Leftrightarrow \operatorname{int}(b d(a))=\emptyset .
\end{aligned}
$$

The class of columnar sets contains more than just open sets and closed sets:

Lemma 7.6 For every topological space $(X, O X)$ and $A \subseteq X$, one has int $(b d(A))=\emptyset$ iff A satisfies the topological McKinsey axiom: $\operatorname{int}(\operatorname{cl}(A)) \subseteq \operatorname{cl}(\operatorname{int}(A)) \Leftrightarrow \operatorname{int}(\operatorname{bd}(A))=$ $\emptyset$.

$$
\text { Proof } \begin{aligned}
\Leftrightarrow \operatorname{int}(\mathrm{cl}(\mathrm{A})) \cap \mathrm{CCintC}(\operatorname{int}(\mathrm{A}))=\emptyset \Leftrightarrow \operatorname{int}(\operatorname{cl}(\mathrm{A})) \cap \mathrm{C}(\operatorname{int}(\mathrm{A}))=\emptyset \\
\Leftrightarrow \operatorname{int}(\mathrm{cl}(\mathrm{A})) \cap \mathrm{C}(\operatorname{int}(\mathrm{A}))=\emptyset \Leftrightarrow \operatorname{int}(\mathrm{bd}(\mathrm{A}))=\emptyset .
\end{aligned}
$$

Recall that set $A \subseteq X$ is nowhere dense iff intcl $(A)=\emptyset$. Closed set $A$ is nowhere dense iff complement CA is dense in X. As is well known, the finite intersection of dense open sets is again dense. Equivalently, the finite union of closed nowheredense sets is still nowhere dense. The following equivalent formulation using these concepts of Lemma 7.6 will be useful:

15 The expression "pedestal" is inspired by the idea that $\mathrm{bd}(\mathrm{A}) \supseteq \mathrm{bd}^{2}(\mathrm{~A})=\mathrm{bd}^{3}(\mathrm{~A})=\ldots=\mathrm{bd}^{\mathrm{n}}(\mathrm{A}) \ldots$ ad infinitum. 
Proposition 7.7 For every topological space (X, OX), one has

$$
\operatorname{bd}(\operatorname{bd}(A))=\operatorname{bd}(A) \text { iff } b d(A) \text { is nowhere dense, i.e., intcl }(b d(A)) \neq \emptyset .
$$

Now, we can prove the following:

Theorem 7.8 For every topological space (X, OX), set MKX of columnar subsets of $X$ is a Boolean algebra.

Proof We already know that $A \in M K X$ iff $C A \in M K X$. Assume $A$ and $B$ to be columnar. Then, $\mathrm{A} \cup \mathrm{B}$ is also shown to be columnar by the following calculation:

$$
\begin{aligned}
\operatorname{bd}(A \cup B) & =\operatorname{cl}(A \cup B) \cap \operatorname{cl}(C(A \cup B))=(\operatorname{cl}(A) \cup \operatorname{cl}(B)) \cap(\operatorname{cl}(C A) \cap \operatorname{cl}(C B)) \\
& \subseteq(\operatorname{cl}(A) \cap(\operatorname{cl}(C A) \cap \operatorname{cl}(C B)) \cup(\operatorname{cl}(B)) \cap(\operatorname{cl}(C A) \cap \operatorname{cl}(C B)) \\
& \subseteq(\operatorname{cl}(A) \cap \operatorname{cl}(C A)) \cup(\operatorname{cl}(B) \cap \operatorname{cl}(C B))=\operatorname{bd}(A) \cup b d(B) .
\end{aligned}
$$

Since $\operatorname{bd}(\mathrm{A})$ and $\mathrm{bd}(\mathrm{B})$ are closed and nowhere-dense sets, their union $b d(A) \cup b d(B)$ is nowhere dense as well, i.e., $\operatorname{int}(b d(A) \cup b d(B))=\varnothing$. Thus, intbd $(A \cup B)=\varnothing$. Similarly, $b d(A \cap B)$ is shown to be nowhere dense by the following calculation using de Morgan's laws for set-theoretical operations $\cap$ and $\cup$ :

$$
\mathrm{bd}(\mathrm{A} \cap \mathrm{B})=\mathrm{bd}(\mathrm{C}(\mathrm{A} \cap \mathrm{B}))=\mathrm{bd}(\mathrm{CA} \cup \mathrm{CB})) \subseteq \mathrm{bd}(\mathrm{CA}) \cup \mathrm{bd}(\mathrm{CB})=\mathrm{bd}(\mathrm{A}) \cup \mathrm{bd}(\mathrm{B})
$$

Hence, if $b d(A)$ and $b d(B)$ are nowhere dense, then $b d(A \cap B)$ is nowhere dense, too. In sum, set MKX of McKinsey sets of $(\mathrm{X}, \mathrm{OX})$ is a Boolean algebra.

Corollary 7.9 Let L be a propositional language with atomic sentences $a, b$... and the usual Boolean connectives $\neg, \wedge, \vee$, and $\rightarrow$, and let $(X, O X)$ be a topological space. Then, a columnar interpretation $L-r \rightarrow M K X$ of $P$ is defined by mapping the atomic sentence $a, b$, onto elements $r(a), r(b), \ldots$ of $M K X$ and interpreting the logical connectives by their set-theoretical counterparts $C, \cap, \cup \ldots$

In sum, if the concept of vagueness is cast in the framework of an S4 operator (such as Bobzien's C), the corresponding boundary operator bd is always stably columnar $\left(b^{3}=b d^{2}\right)$ and quite often even columnar $\left(b^{2}=b d\right)$. One may conjecture that this is because $\mathbf{C}$ satisfies $\mathbf{C} \bullet \mathbf{C}=\mathbf{C}$, i.e., axiom 4 . This is, however, not quite right. As will be shown in the next section, also for Williamson's logic of clarity based on an operator $\mathbf{C}^{*}$ that does not satisfy axiom 4, a well behaved boundary operator bd $\mathrm{BDG}_{\mathrm{BDG}}$ (see Definition 8.6) can be defined that is stably columnar as well.

\section{Columnar Vagueness in Williamson's Logic of Clarity}

In the appendix of Vagueness (Williamson 1994), the author gives a succinct sketch of a modal logic of clarity based on an operator $\mathbf{C}^{*}$ such that $\mathbf{C}^{*} \mathrm{~A}$ is to be read as "It is clearly the case that A". For fixed margin models (the only type of models of the logic of clarity we will consider in the following), $\mathbf{C}^{*}$ defines a modal logic KTB. 
Definition 8.1 The modal logic of clarity operator $\mathbf{C}^{*}$ is defined as an extension of classical Boolean propositional logic by a unary sentential operator $\mathbf{C}^{*}$ such that for all well-formed formulas $\mathrm{A}$ and $\mathrm{B}$, the following expression are valid:

\begin{tabular}{lll}
\hline (1) & $\mathbf{C} *(\mathrm{~A} \longrightarrow \mathrm{B}) \longrightarrow\left(\mathbf{C}^{*} \mathrm{~A} \longrightarrow \mathbf{C}^{*} \mathrm{~B}\right)$ & \\
(2) & (Axiom K) \\
(3) & $\mathrm{CA} \rightarrow>\mathrm{A}$ & (Axiom T) \\
(4) & If $\perp \mathrm{A}$, then $\perp \mathbf{C}^{*} \mathrm{~A}$. & (Axiom B) \\
& & (Rule of \\
& & Necessita- \\
& tion).
\end{tabular}

The details are as follows: We start with a similarity $(\mathrm{W}, \sim)$, where $\mathrm{W}$ is a set (of possible worlds) and relation $\sim$ is a binary similarity relation that is reflexive and symmetric but not necessarily transitive. As usual, sentences A are subsets of W. For $x \in W$, define the similarity neighbourhood $\operatorname{co}(x)$ as $\operatorname{co}(x):=\{y ; x \sim y\}$. Then, we check that for fixed margin models Williamson's clarity operator $\mathbf{C}^{*}$ can be defined as

$$
\mathrm{C}^{*} \mathrm{~A}:=\{\mathrm{x} ; \operatorname{co}(\mathrm{x}) \subseteq \mathrm{A}\}
$$

One observes that $\mathbf{C}^{*}$ in general does not satisfy $\mathbf{C}^{*} \bullet \mathbf{C}^{*}=\mathbf{C}^{*}$ (Axiom 4 ). Nevertheless, it is possible to define a new boundary operator that is related to $\mathbf{C}^{*}$ and is stably columnar. This issue may be of interest to the reader since this new boundary operator is treated neither in Williamson (1994) nor in Williamson (1999). The only boundary operator BD* that is discussed in Williamson (1999, pp. 136-138) is the one that is defined with the aid of $\mathbf{C}^{*}$ in full formal analogy to (3.2):

$$
\text { BD } \left.^{*} \mathrm{~A}:=\operatorname{NOT} \mathbf{C}^{*} \mathrm{~A} \& \operatorname{NOT}\left(\mathbf{C}^{*}(N O T A)\right)\right)
$$

As Williamson rightly remarks, this operator has intuitively rather implausible properties. This holds, in particular, for the behaviour of BD* with respect to iterations. More precisely, the details are as follows. As usual denote the $n$th iteration of the operator $\mathbf{B D}^{*}$ by $\mathbf{B D}^{* \mathrm{n}}$. Then one may ask, whether the operator $\mathbf{B D}^{*}$ is stably columnar in the sense that $\mathbf{B D}^{* \mathrm{n}}=\mathbf{B D}^{* \mathrm{n}+1}$ for some natural number $\mathrm{n} \geq 1$. In Williamson (1999) it is shown by explicit examples that BD* is not stably columnar (see Appendix of Williamson 1999). ${ }^{16}$

The boundary operator to be used in the following is different from BD*. More precisely, we will use a "slim" version of BD* that enjoys better properties. This slim version of BD* was investigated by Breysse and de Glas in detail, see Breysse and De Glas (2007) (without being aware of Williamson's logic of clarity). Their

\footnotetext{
16 Williamson uses the operator BD* to define two concepts of higher order vagueness: A sentence A is said to be $n$ th-order borderline vague if $\mathbf{B D}^{* \mathrm{n}}(\mathrm{A}) \neq \varnothing$. Somewhat more complicated is the definition of $n$ th-order vagueness that uses first order vagueness $\mathbf{B D} *\left(\mathrm{C}_{\mathrm{n}}(\mathrm{A})\right)$ of certain "classifications" $\mathrm{C}_{\mathrm{n}}(\mathrm{A})$ of $A$, $\mathrm{C}_{\mathrm{n}}(\mathrm{A})$ being sets of sentences generated by $\mathrm{A}$ and the operator $\mathrm{C}^{*}$. The relation between $n$ th-order borderline vagueness and $n$ th-order vagueness is complicated.
} 
approach is essentially equivalent to Williamson's approach of fixed margin models based on a similarity structure $(\mathrm{W}, \sim)$ as defined above.

To define the slim version of BD* Breysse and De Glas proceed as follows. Given $(\mathrm{W}, \sim)$, they define operators $\mathrm{PW}-\mathrm{h} \longrightarrow \mathrm{PW}$ and $\mathrm{PW}-\mathrm{s} \longrightarrow \mathrm{PW}$ such that $\mathrm{h}$ is a (non-topological) interior kernel operator and $\mathrm{s}=\mathrm{C} \bullet \mathrm{h} \bullet \mathrm{C}$ is a (non-topological) closure operator. More precisely, $\mathrm{h}$ is just Williamson's operator $\mathbf{C}^{*}$. As is easily seen, $h$ and s satisfy

$$
\mathrm{h}(\mathrm{A})=\{\mathrm{x} ; \operatorname{co}(\mathrm{x}) \subseteq \mathrm{A}\} \quad \mathrm{s}(\mathrm{A})=\{\mathrm{x} ; \operatorname{co}(\mathrm{x}) \cap \mathrm{A} \neq \emptyset\}
$$

Then, operators $\mathrm{h}$ and $\mathrm{s}$ are used to define various concepts of a boundary to be discussed in more detail in the following. To avoid confusing the reader with a mixture of different terminologies and denotations, in the rest of this section, we will use the terminology of Breysse and De Glas (2011). In particular, Williamson's operator C* is denoted by h. Then, we obtain:

Proposition 8.5 Let $(W, \sim)$ be a similarity structure. Then, operators $h$ and $s$ defined above, for all $A \subseteq W$, have the following properties:

(i)

\author{
$h(A) \subseteq A \subseteq S(A)$. \\ $h(A \cap B)=h(A) \cap h(B), s(A \cup B)=s(A) \cup s(B)$. \\ $\operatorname{sh}(A) \subseteq A \subseteq h s(A)$. \\ $h$ is a closure operator; i.e., hs satisfies the \\ Kuratowski axioms (2.1)(2)-(4). ${ }^{17}$ The operator $h$ s \\ is not, however, a topological closure operator; \\ i.e., in general, $h s(A) \cup h s(B)=h s(A \cup B)$ does not \\ hold.
}

As Breysse and De Glas show, operators $h$ and s define a very useful and rich concept of a boundary (cf. Breysse and De Glas 2007, 230ff) as follows:

Definition 8.6 Let $(\mathrm{W}, \sim)$ be a similarity space with operators $h$ and $\mathrm{s}$ as defined above. Then, a boundary operator $\mathrm{bd}_{\mathrm{BDG}}$ can be defined for $\mathrm{A} \subseteq \mathrm{W}$ by

$$
\operatorname{bd}_{\mathrm{BDG}}(\mathrm{A}):=\mathrm{hs}(\mathrm{A}) \cap \mathrm{hs}(\mathrm{CA})=\mathrm{h}(\mathrm{s}(\mathrm{A}) \cap \mathrm{s}(\mathrm{CA})) \text {. }
$$

The operators $b_{\mathrm{BDG}}$ and $\mathbf{B D} *$ are related as follows:

$$
\mathrm{bd}_{\mathrm{BDG}}(\mathrm{A}):=\mathrm{h}(\mathbf{B D} *(\mathrm{~A}))
$$

\footnotetext{
17 The pair (h, s) is well known to be a Galois connection on PW (cf. Denecke et al. 2004). This fact, not observed by Breysse and De Glas, has many interesting consequences, but it is outside the scope of the present paper.
} 
Since $\mathrm{hA} \subseteq \mathrm{A}$, one has $\mathrm{bd}_{\mathrm{BDG}}(\mathrm{A}) \subseteq \mathrm{BD}^{*}(\mathrm{~A})$, and bd $\mathrm{BDG}_{\mathrm{BD}}$ may be intuitively characterized as a slim variant of BD*. While BD* shows a rather erratic behaviour, its slim variant $b_{\mathrm{BDG}}$ behaves much better, as shown by the following theorem:

Theorem 8.8 Operator $b d_{B D G}$ defined by Definition 8.6 is stably columnar for all $A \subseteq W$ :

$$
\operatorname{bd}_{\mathrm{BDG}}\left(\operatorname{bd}_{\mathrm{BDG}}\left(\operatorname{bd}_{\mathrm{BDG}}(\mathrm{A})\right)\right)=\operatorname{bd}_{\mathrm{BDG}}\left(\operatorname{bd}_{\mathrm{BDG}}(\mathrm{A})\right) \text {. }
$$

Proof The proof is carried out by using (8.2)(i)-(iv) and some other familiar properties of closure operators. By definition,

$$
\begin{aligned}
\operatorname{bd}_{\mathrm{BDG}}\left(\operatorname{bd}_{\mathrm{BDG}}\left(\operatorname{bd}_{\mathrm{BDG}}(\mathrm{A})\right)\right) & =\mathrm{hs}\left(\operatorname { b d } _ { \mathrm { BDG } } ( \operatorname { b d } _ { \mathrm { BDG } } ( \mathrm { A } ) ) \cap \mathrm { hs } \left(\operatorname{Cbd}_{\mathrm{BDG}}\left(\operatorname{bd}_{\mathrm{BDG}}(\mathrm{A})\right)\right.\right. \\
& =\operatorname{bd}_{\mathrm{BDG}}\left(\operatorname{bd}_{\mathrm{BDG}}(\mathrm{A})\right) \cap \mathrm{Csh}\left(\operatorname{bd}_{\mathrm{BDG}}\left(\operatorname{bd}_{\mathrm{BDG}}(\mathrm{A})\right) .\right.
\end{aligned}
$$

If we can prove that $\mathrm{Csh}\left(\mathrm{bd}_{\mathrm{BDG}}\left(\mathrm{bd}_{\mathrm{BDG}}(\mathrm{A})\right)=\mathrm{W}\right.$, we are done. This is carried out as follows:

$$
\begin{aligned}
\operatorname{Csh}\left(\operatorname{bd}_{\mathrm{BDG}}\left(\operatorname{bd}_{\mathrm{BDG}}(\mathrm{A})\right)=\right. & \mathrm{W} \Leftrightarrow \operatorname{sh}\left(\mathrm{bd}_{\mathrm{BDG}}\left(\mathrm{bd}_{\mathrm{BDG}}(\mathrm{A})\right)=\emptyset \Leftrightarrow \mathrm{h}\left(\mathrm{bd}_{\mathrm{BDG}}\left(\operatorname{bd}_{\mathrm{BDG}}(\mathrm{A})\right)=\emptyset\right.\right. \\
& \Leftrightarrow \mathrm{h}\left(\mathrm { hs } \left(\operatorname{bd}_{\mathrm{BDG}}(\mathrm{A}) \cap \mathrm{hs}\left(\operatorname{Cbd}_{\mathrm{BDG}}(\mathrm{A})\right)=\emptyset\right.\right. \\
& \Leftrightarrow \mathrm{h}\left(\operatorname{bd}_{\mathrm{BDG}}(\mathrm{A}) \cap \mathrm{hCh}\left(\mathrm{bd}_{\mathrm{BDG}}(\mathrm{A})\right)=\emptyset\right. \\
& \left.\Leftrightarrow \operatorname{hbd}_{\mathrm{BDG}}(\mathrm{A})\right) \cap \mathrm{hCh}\left(\mathrm{bd}_{\mathrm{BDG}}(\mathrm{A})\right)=\emptyset .
\end{aligned}
$$

Clearly hbd $\left.\mathrm{BDG}(\mathrm{A})) \cap \mathrm{hCh}\left(\mathrm{bd}_{\mathrm{BDG}}(\mathrm{A})\right) \subseteq \operatorname{hbd}_{\mathrm{BDG}}(\mathrm{A})\right) \cap \mathrm{Ch}\left(\mathrm{bd}_{\mathrm{BDG}}(\mathrm{A})\right)=\varnothing$.

In sum, both Bobzien's S4 logic of clearness based on $\mathbf{C}$ and Williamson's nonS4 logic of clarity for fixed margin models based on $\mathbf{C}^{*}$ give rise to stably columnar boundary operators bd and $\mathrm{bd}_{\mathrm{BDG}} \cdot{ }^{18}$

\section{Concluding Remarks}

If one relies on topological, i.e., $\mathbf{S} 4$ models of modal operators $\mathbf{C}$ and $\mathbf{U}$, columnar vagueness crops up in more places than is usually assumed, be it in its strict version (i.e., for all subsets of weakly scattered spaces, or at least for all columnar sets of arbitrary spaces) or in a weaker form as stably columnar vagueness for all subsets of all topological spaces. ${ }^{19}$ This entails, in particular, that vague concepts, when represented by open subsets of conceptual spaces, are columnar. An analogous result holds for fixed margin models $(\mathrm{W}, \sim)$ that lead to a KTB logic of the modal operator $\mathbf{C}^{*}$.

\footnotetext{
18 As Williamson points out, the logic of variable margin models does not satisfy the Brouwer axiom (B) (cf. Williamson 1994, 272). As is easily calculated, this entails that the operator hs is no longer a closure operator.

${ }^{19}$ Examples of such topology-affine approaches to vagueness dealt with in this paper are that of Bobzien, Rumfitt, Williamson, Sainbury, and Wright.
} 
Thus, if one rejects columnar vagueness for one philosophical reason or other, one must reject S4 or KTB models of vagueness altogether. On the other hand, if one accepts an S4 approach for the clearness operator $\mathbf{C}$ or a KTB approach for the clarity operator $\mathbf{C}^{*}$, one must buy into columnar vagueness for the resulting concept of a (slim) boundary at least if one restricts one's consideration to classical modal logic, i.e., normal modal logic. The situation may be different if one gives up normality. However, this is not an issue to be treated in this paper.

Acknowledgements I thank Nasim Mahoozi for many intense and detailed discussions on matters of vagueness, topology, and related issues that greatly helped me improve my understanding of the topics dealt with in this paper. Further I would like to thank an anonymous referee for very insightful and constructive criticisms on a previous version of this paper. Financial support of the Ministerio de Economía y Competividad (MINECO) del Gobierno Español, project "Representación y anticipación: modelización interventiva RRI en las ciencias y técnicas emergentes", is gratefully acknowledged.

\section{References}

Alexandroff, P. (1937). Diskrete Räume. Rec. Math. [Mat. Sbornik] N.S., 2(44)(3), 501-519.

Baltag, A., Bezhanishvili, N., Özgün, A, Smets, S. (2018). A topological approach to full belief. Journal of Philosophical Logic, 48(2), 205-244.

Bezhanishvili, G., Esakia, L., \& Gabelaia, D. (2004). Modal Logics of submaximal and nodec spaces, Festschrift for Dick de Jongh on his 65th birthday, University of Amsterdam.

Bezhanishvili, G., Mines, R., \& Morandi, P. J. (2003). Scattered, Hausdorff-reducible, and hereditarily irresolvable spaces. Topology and Its Applications, 132, 291-306.

Bobzien, S. (2010). Higher-order vagueness, radical unclarity and absolute agnosticism. Philosophers' Imprint, 10(10), 1-30.

Bobzien, S. (2012). If it's clear, then it's clear that it's clear, or is it? Higher-order vagueness and the S4 axiom. In K. Ierodiakonou \& B. Morison (Eds.), Episteme, etc: Essays in honour of Jonathan Barnes (pp. 189-212). Oxford: Oxford University Press.

Bobzien, S. (2013). Higher-order vagueness and borderline nestings: A persistent confusion. Analytic Philosophy, 54(1), 1-43.

Bobzien, S. (2015). Columnar higher-order vagueness, or vagueness is higher-order vagueness. Proceedings of the Aristotelian Society Supplementary, 89, 61-89.

Bobzien, S., \& Rumfitt, I. (2019). Intuitionism and the modal logic of vagueness. Journal of Philosophical Logic (to appear).

Breysse, C., \& De Glas, M. (2007). A new approach to the concepts of boundary and contact: Toward an alternative to mereotopology. Fundamenta Informaticae, 78, 217-238.

Denecke, K., Erné, M., \& Wismath, S. L. (Eds.). (2004). Galois connections and applications. Dordrecht: Kluwer.

Dunn, J. M., \& Hardegree, G. M. (2001). Algebraic methods in philosophical logic, Oxford logic guides 41. Oxford: Clarendon Press.

Gabelaia, D. (2001) Modal definability in topology. Master's Thesis, Institute for Logic, Language and Computation (ILLC), University of Amsterdam.

Gärdenfors, P. (2000). Conceptual spaces. Cambridge, MA: MIT Press.

Gärdenfors, P. (2014). The geometry of meaning: Semantics based on conceptual spaces. Cambridge, MA: MIT Press.

Heck, R. G., Jr. (1993). A note on the logic of (higher-order) vagueness. Analysis, 53, 201-209.

McKinsey, J. C. C., \& Tarski, A. (1944). The algebra of topology. Annals of Mathematics, 45, 141-191.

Mormann, T. (2013). Heyting mereology as a framework for spatial reasoning. Axiomathes, 23(1), $137-164$.

Rumfitt, I. (2015). Boundary stones of thought. Oxford: Oxford University Press.

Sainsbury, M. (1990). Concepts without boundaries, inaugural lecture at the University of London. Page references are to the reprint in Keefe and Smith (eds.) (1996, pp. 251-64). 
Tarski, A. (1938). Sentential calculus and topology. In A. Tarski (1956) Logic, semantics, metamathematics, papers from 1923 to 1938, 421 to 454. Oxford: OUP.

van Benthem, J., \& Bezhanishvili, G. (2007). Modal logics of space. In M. Aiello, I. Pratt-Hartmann, \& J. van Benthem (Eds.), Handbook of spatial logics (pp. 217-298). Berlin: Springer.

Williamson, T. (1994). Vagueness. London, New York: Routledge.

Williamson, T. (1999). On the structure of higher-order vagueness. Mind, 108, 127-142.

Wright, C. (1992). Is higher order vagueness coherent? Analysis, 52(3), 129-139.

Wright, C. (2007). Wang's paradox. In R. E. Auxier \& L. E. Hahn (Eds.), The philosophy of Michael Dummett. Chicago, LaSalle: Open Court Publishing Company.

Zardini, E. (2006). Higher-order vagueness and paradox: The glory and misery of S4-definiteness. The Baltic International Yearbook of Cognition, Logic, and Communication, 1, 203-220.

Zarycki, M. (1927). Quelques notions fondamentales de I'Analysis Situs du point de vue de 1'Algébre de la Logique. Fundamenta Mathematicae, 9, 3-15.

Zenker, F., \& Gärdenfors, P. (2015). Applications of conceptual spaces. The case for geometric knowledge representation. Berlin: Springer.

Publisher's Note Springer Nature remains neutral with regard to jurisdictional claims in published maps and institutional affiliations. 